\title{
Optical Cavity Implementations of the Quantum Walk
}

\author{
Peter L. Knight, Eugenio Roldán†, and J. E. Sipe \\ Optics Section, Blackett Laboratory, Imperial College London, \\ London SW7 2AZ, United Kingdom
}

November 9, 2018

\begin{abstract}
We discuss how the coined quantum walk on the line or on the circle can be implemented using optical waves. We propose several optical cavity configurations for these implementations.

PACS: 03.67.Lx, 05.40.Fb
\end{abstract}

\section{Introduction}

Classical implementations of quantum computing algorithms are interesting for both practical and fundamental reasons. They allow actual implementations that may serve as model systems for their quantum counterparts, and they may shed some light on the profound differences between classical and quantum waves.

The concept behind these classical implementations is simple: waves share with quantum systems the possibility of creating superposition states [1]. Of course, the entanglement of separated systems that is specific to quantum mechanics is not present, but such entanglement does not seem to play a central role in at least some of the proposed quantum computing algorithms. This subject has been considered by a number of authors 1-4. In particular, implementations of Grover's quantum search algorithm have been proposed [3], and actually implemented [4, using only classical resources. It must be pointed out however that the lack of entanglement in the classical case manifests through the exponential increase of the ammount of classical resources needed with the increase of the problem size [1], and it would be more appropriate to speak

${ }^{*}$ E-mail: p.knight@imperial.ac.uk

${ }^{\dagger}$ Permanent address: Departament d'Òptica, Universitat de València, Dr. Moliner 50, 46100-Burjassot, Spain. E-mail: eugenio.roldan@uv.es

${ }^{\ddagger}$ Permanent address: Department of Physics, University of Toronto, Toronto M5S 1A7, Canada. E-mail: sipe@physics.utoronto.ca 
of simulations rather than actual implementations, we shall come back to this point later.

The so-called quantum random walk (QW in the following) is a process that has received a lot of attention in the quantum computing community [5]-20]; see the recent review by Julia Kempe [21. The QW was introduced by Aharonov et al. [5] ten years ago as the quantum analog of the classical random walk (RW for short). The attention devoted to QWs is mainly motivated by the fact that as some problems can be solved efficiently in classical computation with algorithms based on RWs, there is a reasonable hope that QWs could give rise to algorithms faster than classical for some specific problems. In work along these lines, Kempe [10] showed that the hitting time of the discrete QW from one corner of an $n$ bit hypercube to the opposite corner is polynomial in the number of steps, $n$, whilst it is exponential in $n$ in the classical case. Subsequently Shenvi et al. 14] showed that a QW can perform the same tasks as Grover's search algorithm, and Childs et al. [15] have introduced an algorithm for crossing a special type of graph exponentially faster than can be done clasically. Moreover, as classical RWs are used in proofs of complexity to place bounds on the difficulty of certain problems, then one might hope for similar proofs for quantum algorithms using QWs [22].

QWs can be performed by a quantum computer, and a number of possible implementations have been proposed: Travaglione et al. 23. proposed its implementation in an ion trap quantum computer, Dür et al. [24] consider the QW in an optical lattice, and Sanders et al. 25] have suggested its experimental realization in cavity quantum electrodynamics. All these proposals consider quantum systems. A different approach was followed by Zhao et al. [26, and subsequently by Jeong et al. [27, who discussed the implementation of QWs using single photons and linear optical elements. But it turns out that the quantum nature of light does not play any role in the developement of the walk. That is, QWs can be performed within classical optics. This has been noticed by Hillery et al. [18] who have developed an interferometric analogy of QWs and made a suggestion similar to that of Refs. 26, 27. The problem with these last proposals is that the number of optical elements grows quickly with the number of steps in the QW.

In a recent paper 28 we have shown that QWs can be understood as an interference phenomenon (and thus as a classical phenomenon in the sense that quantization does not play any role in its understanding) and can be clasically implemented in optical cavities. The advantage of the optical cavity approach is that the resources needed (in the sense of the number of optical elements) do not depend on the size of the implemented walk. Moreover, we argued that the coined QW on the line had indeed been optically implemented by Bouwmeester et al. 29] in the context of an optical implementation of the Galton board, although the authors did not explicitly make this link.

In the present paper we extend the results advanced in [28] by considering new cavity configurations, by proposing schemes for the implementation of the QW on the circle, and by discussing in detail up to what extent the experiment of Bouwmeester et al. [29] constitutes an actual implementaion of the QW. 


\section{The coined quantum walk}

In order to make this paper as self-contained as possible we briefly review here some of the main characteristics of QWs.

Let us first consider the classical RW on the line. In a common version the "walker" - the particle or system performing the RW - takes one step to the right or to the left depending on the (random) result of tossing a coin. After $n$ steps, the probability of finding the walker at a distance $m$ from the origin is given by the binomial distribution which, for large $n$, is well approximated by a Gaussian (dashed line in Fig. 1) whose standard deviation is given by $\sqrt{n}$.

In the coined QW, the role of the coin is played by a qubit, that is, by a two-level system. In analogy with its classical counterpart, the quantum walker moves to the right or to the left depending on the internal state of the qubit. The obvious difference with the classical RW is that the coin-qubit can be set in a superposition state, and thus the walker can be described by probability amplitudes associated with movement both to the right and to the left. After each displacement of the walker, the state of the qubit is set to a superposition state by means of a suitable unitary transformation, typically (but not necessarily) the Hadamard transformation. This operation plays, in a sense, the role of the toss of the coin in the RW. Then a new displacement occurs, and so on and so forth.

Figure 1: Probability distribution for $n=200$ for both the classical random walk (dashed) and quantum walk (continuous). The initial conditions chosen for calculating the QW were $R_{0,0}=1 / \sqrt{2}$ and $L_{0,0}=i / \sqrt{2}$, see Eqs.(1112). Notice that the quantum $P_{m}$ is null for odd $m$ at odd $n$. We have represented only nonzero values and joined them to guiding the eye.

The probability distribution in the quantum case is very different from the classical one in several respects. It resembles the Airy function, as can be seen in Fig.1 and we discuss elsewhere the asymptotics of the walk distribution in terms of Airy functions [28. The standard deviation of the QW probability distribution is linear in $n$; that is, the quantum walker walks quadratically faster than the classical walker. With increasing time steps, the probability distribution 
is null for alternatively even and odd values of $m$. Except for these nulls, the probability distribution is quite flat and uniform in its central region. Finally, we point out that the form of the probability distribution depends strongly on the initial state of the coin. Several analytical approaches have been developed that provide explicit solutions of the QW on the line [6, 8, 19.

The QW on the circle is similar to the $\mathrm{QW}$ on the line. But the circle contains only a finite number of discrete points from $m=0$ to $m=M-1$ and then position $m=M+1$ is the same as position 0 and so on. The probability distribution is, of course, very different to that of the QW on the line (it does not converge to a limit), and in Fig.2 we have plotted three examples that are detailed in the caption (see also, e.g., 9, 17]).

Figure 2: Probability distribution for the indicated number of steps, $n$, for the quantum walk on a circle with 61 discrete positions. The initial is $R_{30,0}=1 / \sqrt{2}$ and $L_{30,0}=i / \sqrt{2}$.

So-called quantum random walks are of course not random, since the evolution of the quantum walk is completely deterministic, and randomness enters only when a position measurement of the walker is finally performed. Thus we refer to this process simply as a "quantum walk." We mention that a different but related type of quantum walk, the so-called continuous QW, was introduced 
by Farhi and Gutmann [7. The continuous QW does not need the use of any coin or qubit. We refer the interested reader to the literature [7, 21] for the details of this walk, which we will not consider here.

\section{Classical implementation of the quantum walk in optical cavities}

\subsection{The Hadamard walk}

Let us first consider the implementation of the standard Hadamard walk. In our approach, the role of the walker will be played by the frequency of a light field, and the role of the coin will be played by some other degree of freedom of the light field. This can be its spatial path or its polarization state. That is, in the terminology of [1, we consider both position and polarization cebits. A cebit is a two-component complex vector representing the light field, and is the classical counterpart of the qubit.

For example, the light field

$$
\vec{E}=\sum_{m=-l}^{l} \vec{E}_{m} e^{i\left[\left(\omega_{0}+m \bar{\omega}\right) t-k_{m} z\right]}+c . c .,
$$

where $\omega_{0}$ is the carrier frequency, $\bar{\omega}$ is the frequency difference between successive frequency components, and $z$ is the propagation direction (the vector $\vec{E}_{m}$ lies in the $(x, y)$ plane), can be represented by the abstract state

$$
\left.\left.|\psi\rangle=\sum_{m=-l}^{l}\left[R_{m} \mid m, x\right)+L_{m} \mid m, y\right)\right]
$$

where $R_{m} \equiv \hat{x} \cdot \vec{u}_{m}, L_{m} \equiv \hat{y} \cdot \vec{u}_{m}\left(\vec{u}_{m}=\vec{E}_{m} /\left|\vec{E}_{m}\right|\right)$ and $\sum_{m=-l}^{l}\left[\left|R_{m}\right|^{2}+\left|L_{m}\right|^{2}\right]$ $=1$. In this notation the basis vectors $\mid m, c)$ label the frequency and polarization, with $c=x, y$ and we can associate $x(y)$ with the coin head (tail). We can also consider position (or direction) cebits, that is, a field of the form

$$
\vec{E}=\sum_{c=1,2} \sum_{m=-l}^{l} \vec{e}_{c} E_{m, c} e^{i\left[\left(\omega_{0}+m \bar{\omega}\right) t-k_{m} r_{c}\right]}+c . c .,
$$

where $r_{c}=\hat{k}_{c} \cdot \vec{r}\left(\hat{k}_{c}\right.$ denotes the direction of propagation of the $c^{\text {th }}$ field), and $\vec{e}_{c}$ denotes its polarization. This can be represented by the state

$$
\left.\left.|\psi\rangle=\sum_{m=-l}^{l}\left[R_{m} \mid m, r_{1}\right)+L_{m} \mid m, r_{2}\right)\right],
$$

where now $R_{m} \equiv E_{m, 1} /\left(\left|E_{m, 1}\right|^{2}+\left|E_{m, 2}\right|^{2}\right)$ and $L_{m} \equiv E_{m, 2} /\left(\left|E_{m, 1}\right|^{2}+\left|E_{m, 2}\right|^{2}\right)$. Generally we use the index $c$ in $\mid m, c)$ to label the variable that plays the role 
of the internal state of the coin; this is the polarization $c=x, y$ in our first example, and the different paths, $c=r_{1}, r_{2}$, in our second. Of course more than two optical paths can be considered, and thus coin-cebits with more that two components; this would be useful for the implementation of multidimensional QWs [13, but we shall not consider these more complication dynamics here.

As stated, the walk is executed by the light frequency. Then there must be a unitary operator that performs the conditional displacement operation

$$
\left.\left.\hat{V} \mid m, c_{i}\right)=\mid m \pm 1, c_{i}\right), \quad i=1,2 .
$$

For polarization cebits, $\hat{V}$ can be physically implemented with an electrooptic modulator $(\mathrm{EOM})$ to which a linearly time dependent voltage is applied: the $x$ $(y)$ polarization component of the field frequency component $(\omega+n \bar{\omega})$ will see its frequency increased (decreased) by an ammount $\bar{\omega}$. For a position cebit, the EOMs must be adjusted so they are not polarization selective. Two such EOMs are needed, one to increase the frequencies in one of the beams, and the second to decrease the frequencies in the other beam.

After each displacement of the field frequency, a Hadamard transformation acting on the cebit has to be implemented. The Hadamard transformation is represented by the operator

$$
\hat{H}=\frac{1}{\sqrt{2}}\left(\begin{array}{cc}
1 & 1 \\
1 & -1
\end{array}\right),
$$

whose action on the cebits reads

$$
\begin{aligned}
& \left.\left.\left.\hat{H} \mid m, c_{1}\right)=\frac{1}{\sqrt{2}}\left[\mid m, c_{1}\right)+\mid m, c_{2}\right)\right], \\
& \left.\left.\left.\hat{H} \mid m, c_{2}\right)=\frac{1}{\sqrt{2}}\left[\mid m, c_{1}\right)-\mid m, c_{2}\right)\right] .
\end{aligned}
$$

For polarization cebits, the Hadamard transformation is optically implemented using a half-wave plate (HWP) with its fast axis forming an angle $\pi / 8$ with respect to the $\hat{x}$ axis. For position cebits, it is implemented using a beamsplitter with two $\pi / 2$ phase shifters [1, 2].

The QW is implemented by the repeated action on the cebit of the operator $\hat{H} \hat{V}$, that is, after $n$ iterations the state reads

$$
\left.\mid \psi)_{n}=[\hat{H} \hat{V}]^{n} \mid \psi\right)_{0},
$$

that can be written as

$$
\left.\left.\mid \psi)_{n}=\sum_{m=-n}^{+n}\left[R_{m} \mid m, c_{1}\right)+L_{m} \mid m, c_{2}\right)\right],
$$

with

$$
\begin{aligned}
& R_{m, n}=\frac{1}{\sqrt{2}}\left[R_{m-1, n-1}+L_{m+1, n-1}\right], \\
& L_{m, n}=\frac{1}{\sqrt{2}}\left[R_{m-1, n-1}-L_{m+1, n-1}\right],
\end{aligned}
$$


where $R_{m, 0}=L_{m, 0}=0$, if $m \neq 0$ and $R_{m,-1}=L_{m,-1}=0 \forall m$. Finally, the intensity of each frequency component of the light field, which is the optical analog of the probability of finding the walker at position $m$ at iteration (time) $n$, is given by

$$
P_{m}(n)=\left|R_{m, n}\right|^{2}+\left|L_{m, n}\right|^{2},
$$

which is represented in Fig. $1^{1}$.

In order to implement $n$ steps the best option is to introduce the described elements in an optical cavity. In the next subsection we propose several designs for optical cavities in which the QW on the line is performed.

\subsection{Optical cavities for the QW on the line}

An optical cavity imposes the constraint that the optical frequencies must fit within a set of eigenfrequencies defined $e . g$. by the length of the cavity. Thus, the time dependent electric field applied to the EOMs and the cavity length must be adjusted in such a way that the frequency shift $\bar{\omega}$ matches the cavity free spectral range. We discuss this important point below. It is also important to notice that the field injected in the cavity from the outside (the initial state) can be injected only once to effect the kind of initial conditions described above; more complicated walks would be generated from multiple injections. In our analysis we assume there are negligible cavity losses; in practice such losses would limit the number of steps that could be implemented, unless an amplifier were added to the cavity to compensate them. In Figs. 3 and 4 we illustrate in schematic form several possible designs for the implementation of the QW on the line.

In Figs.3(a) and 3(b) a polarization cebit is considered. In the scheme of Fig.3(a) we represent a ring cavity containing an EOM and a HWP, as discussed in the previous section. In the scheme of Fig.3(b) the concept is the same but utilizes a linear cavity. As a result, (i) the HWP has to be replaced by a quarter wave-plate, QWP, and (ii) the frequency shift introduced by the EOM must be half the size as compared to the ring cavity, as light crosses both elements twice in every roundtrip.

Let us discuss in more detail the value of the frequency jump $\bar{\omega}$. We have commented above that the cavity imposses that $\bar{\omega}$ fits the cavity free spectral range $(F S R)$, but the frequency shift introduced by the EOM cannot be better resolved than the inverse of the cavity roundtrip time $\tau_{c}$, which is precisely $F S R$. Then the steps of the QW would not be well resolved if their size equals $F S R$. Nevertheless there are several ways of overcoming this difficulty. One way is to take the frequency jump equal to several free spectral ranges, i.e., $\bar{\omega}=f$ $F S R$ with $f$ an integer larger than one. In this way the uncertainty in the frequency displacement does not avoid the resolution of the QW steps. Another possibility is that the frequency shift introduced by the EOM be smaller than

\footnotetext{
${ }^{1}$ We notice that the QW can also be defined as the repeated action of the operator $\hat{V} \hat{H}$ as in 8 . In this case the probability distribution is the same but the equations of evolution of the amplitudes are different although can be easily related to the ones used here.
} 
$F S R$. In this case it would be necessary several roundtrips to perform a single frequency step of the QW and then it would be necessary to control the action of the HWP (or the QWP) that performs the Hadamard transformation as it should not act until the frequency step of the QW is completed. For example, if the frequency step takes five cavity roundtrips, the HWP should act only once every five rountrips. This can be accomplished by substituting the HWP by a second EOM to which a constant voltage of appropriate magnitude is applied, this voltage acting only every five cavity roundtrips. There is still another possibility by using a single pulse light field with a pulse duration shorter than $\tau_{c}$. In this case $\bar{\omega}$ should be larger than the pulse spectral width but it would be not necessary to match an integer of FSR. In any case, it is clear that the sequence of frequency displacement and Hadamard transformation can be performed within an optical cavity with enough resolution.

Figure 3: Schemes for the optical implementation of the QW on the line using polarization cebits. In (a), a ring cavity, the electrooptic modulator (EOM) shifts up (down) the frequency of the $x(y)$ polarization component of the light field in $\bar{\omega}$, and a half-wave plate (HWP) with its axis forming an angle $\pi / 8$ with respect to the $x$-axis, performs the Hadamard transformation. In (b), a linear cavity, the EOM modulator shifts the field frequency $\bar{\omega} / 2$ depending on the field frequency as in (a), and a quarter-wave plate (QWP) performs the Hadamard transformation (notice that in the Fabry-Perot cavity the light passes twice through each intracavity element every roundtrip).

In the ring cavity shown in Fig.4(a), a position cebit is used. Two electrooptic modulators, EOM1 and EOM2, are needed here, each one of them acting on each of the two sides of the cavity, which play the role of the two coin states. The action of these EOMs must be polarization independent. Alternately, the polarization of the light must be aligned with the axes of the EOMs, and the set of axes of one EOM must be aligned with the set of axes of the other. One of the EOMs increases the field frequency by $\bar{\omega}$, while the other decreases it by the same amount. The Hadamard transformation is performed by the beamsplitter BS; we do not represent in the figure the additional phase filters necessary for making a Hadamard, [1, 2].

In Fig.4(b), again a ring cavity, a hybrid between a polarization and a direction cebit is considered. In this bidirectional cavity the horizontal and vertical polarization components of the field are forced to travel in counterpropagating 
Figure 4: Schemes for the optical implementation of the QW on the line using position cebits. In (a), two coupled unidirectional ring cavities, EOM1 and EOM2 increase and decrease, respectively, the field frequency every roundtrip and the Hadamard transformation is performed by the beamsplitter BS. The bidirectional ring cavity in (b) is designed for sustaining a $x(y)$ polarized field in the clockwise (counterclockwise) direction. The EOM increases (decreases) the $x(y)$ polarized field frequency and the set formed by the two quarter-wave plates QWP1 (axis $\pi / 4$ ), QWP2 (axis $-\pi / 4$ ), and BS performs the Hadamard transformation, see text.

directions. As in the cases described in Fig.3, a single EOM is needed that increases (decreases) the field frequency of the $x(y)$ polarization component in $\bar{\omega}$. The set of devices formed by the two quarter-wave plates QWP1 (axis oriented at $\pi / 4$ ) and QWP2 (axis oriented at $-\pi / 4$ ) and the BS plays a double function: (i) it guarantees that the clockwise (counterclockwise) field remains $x(y)$ polarized, and (ii) it performs the Hadamard transformation. The Hadamard transformation is implemented by the BS; when done in the presence of the two QWP, this guarantees that the transmitted field maintains its polarization, while the reflected field changes its polarization from $x$ to $y$ and vice versa. Here is how it works: Note that when traversing the three elements the polarization of a beam does not change, since the effect of QWP1 is cancelled out by the effect of QWP2, and vice versa. For light reflected by the BS, on the other hand, QWP1 (or QWP2) is crossed twice, which is equivalent to the effect of a HWP with axis at $\pi / 4(-\pi / 4)$; this changes $x$ polarization to $y$ polarization (or $y$ to $x$ ). 
An interesting aspect of the two schemes shown in Fig.4 is that the output corresponds to only one of the two cebit states and thus the spectrum of the light does not correspond to $P_{m}(n)$, Eq.(13), but to $P_{m}^{R}(n)=\left|R_{m, n}\right|^{2}$ or $P_{m}^{L}(n)=$ $\left|L_{m, n}\right|^{2}$. In order to obtain the complete QW, one should allow the light to exit the cavity through two of the cavity mirrors and then combine the two beams into a single beam. In Fig.5 we represent $P_{m}(n)$ together with $P_{m}^{R}(n)$ and $P_{m}^{L}(n)$ for an initial condition different to that of Fig.1, see caption.

Figure 5: Probability distribution for $n=200$ for the quantum walks. The initial conditions chosen for calculating the QW were $R_{0,0}=1$ and $L_{0,0}=0$, see Eqs. (1112). Notice that $P_{m}$ is null for odd $m$ at odd $n$. We have represented only nonzero values. $P_{m}^{x}, P_{m}^{y}$, and $P_{m}$ are represented in (a), (b), and (c), respectively.

\subsection{More general walks}

The Hadamard walk is only one of several possible ways of performing a QW. What is actually needed is a unitary transformation that mixes the two coincebit states after each system displacement but it is not necessary that this unitary transformation be the Hadamard transformation. That is, there is a family of unitary transformations that provide the same final probability distribution, (see, e.g., 8, 11, 12]). In particular, Konno [1] has studied QWs for 
which the unitary transformation applied to the qubit after each displacement reads

$$
U=\left(\begin{array}{ll}
a & b \\
c & d
\end{array}\right),
$$

where $a, b, c$ and $d$ are complex, in general, and satisfy

$$
\begin{aligned}
|a|^{2}+|b|^{2} & =|c|^{2}+|d|^{2}=1, \\
a c^{*}+b d^{*} & =0, \\
|\Delta| & =1, \Delta=a d-b c, \\
c & =-\Delta b^{*}, d=\Delta a^{*} .
\end{aligned}
$$

Konno 11] demonstrated that, after a large number of iterations $n$, for QWs governed by such a $U$ the mean displacement of the walker, $\langle x\rangle$, and the mean quadratic displacement, $\left\langle x^{2}\right\rangle$, are given by

$$
\begin{aligned}
\langle x\rangle & =\left[|\beta|^{2}-|\alpha|^{2}+\frac{2 \operatorname{Re}\left(a b^{*} \alpha \beta^{*}\right)}{|a|^{2}}\right] \\
\cdot & (1-|b|) n, \\
\left\langle x^{2}\right\rangle & =(1-|b|) n^{2},
\end{aligned}
$$

where $\mid c)_{0}=\operatorname{col}(\alpha, \beta)$ is the initial state of the coin-qubit. The above results apply, obviously, to the Hadamard walk by taking $a=b=c=-d=1 / \sqrt{2}$.

For the implementations of the QW here considered, this means that it is not essential that the unitary transformation after each frequency displacement be implemented by a HWP, in the case of polarization cebits, nor by a 50/50 beamsplitter for position cebits, as they can be substituted by any unitary transformation of the type $U$ on the polarization or position. Now we have the elements required for discussing the experiemnt of Ref. [29].

\subsection{The experiment of Bouwmeester et al. [29]}

Bouwmeester 29] proposed an optical implementation of the Galton board (the quincunx), and studied it both theoretically and experimentally. What was actually implemented is a grid of Landau-Zener crossings through which a light beam propagates, and the focus was the study of the appearance of recurrences in the spectrum of the light field. We refer the interested reader to Ref. [29] for details.

A simplified version of the experimental device used in [29] is that represented in Fig.3(b), but replacing the QWP by a second EOM with its axis rotated $\pi / 4$ with respect to the axis of the first EOM. The second EOM introduces a dephasing $\delta / 2$ between the two polarization components, where $\delta$ can be varied at will. Then, after a cavity roundtrip, the matrix representing the

action of the second EOM action on the $x$ and $y$ polarization components of the field reads

$$
U_{\delta}=\left(\begin{array}{cc}
\cos \delta & -i \sin \delta \\
-i \sin \delta & \cos \delta
\end{array}\right) .
$$


It is easy to see that $U_{\delta}$ can be seen as a particular case of $U$ (14) for $a=d=$ $\cos \delta$ and $b=c=-i \sin \delta$. From Eqs.(16) and (17) we find

$$
\begin{aligned}
\langle x\rangle & =\left[|\beta|^{2}-|\alpha|^{2}+2 \operatorname{Im}\left(\alpha \beta^{*}\right) \tan \delta\right] \\
\cdot & (1-\sin \delta) n, \\
\left\langle x^{2}\right\rangle & =(1-\sin \delta) n^{2} .
\end{aligned}
$$

Notice that for $\alpha=\beta$ both real quantities - that is, for an initial linearly polarized state $-\langle x\rangle=0$ for all $\delta$ and the variance of the spectrum is $\sigma=$ $\sqrt{1-\sin \delta}$.

From this it is clear that the experimental device used by Bouwmeester 29] can implement QWs. In fact, in Fig.6 of their work [29], which corresponds to $\delta=\pi / 5$, a power spectrum very similar to those expected for the optical implementation of the QW can be clearly appreciated. The authors [29] considered Fig.6 in their manuscript as a demonstration of the coherence quality of their system, and did not make an explicit connection of their work with QWs.

Nevertheless there is subtle but essential difference between the experiement of 29] and an actual implementation of the QW. In 29 the frequency displacement $\bar{\omega}$ is a fraction of the FSR but the unitary transformation (18) is applied at every roundtrip (see our discussion above in Subsection 3.2). Then, the experiment in 29] cannot be regarded as an implementation of the QW although is something certainly very similar. That is, the optical Galton board is different from the QW. Nevertheless we can consider the experiment of Bouwmeester et al. 29] as a proof of principle that the QW can be implemented in an optical cavity.

\subsection{Quantum walk on the circle}

The QW on the circle, again with the frequency playing the role of position, can be also implemented optically. If $2 M-1$ is the number of possible discrete values for the frequency, it is necessary to devise a way of shifting the frequency $\omega+(M+1) \bar{\omega}$ to $\omega-M \bar{\omega}$ and the frequency $\omega-(M+1) \bar{\omega}$ to $\omega+M \bar{\omega}$. This can be done by substituting the EOMs in the schemes of Figs. 3 and 4 by the device shown in Fig.6, which we denote as $\overline{E O M}$. It consists of two electrooptic modulators and two specially designed mirrors. Mirrors M1 and M2 only reflect the frequencies $\omega+M \bar{\omega}$ and $\omega-M \bar{\omega}$, respectively, and are transparent to the rest of the frequencies in the spectrum.

To see how the circle walk results, we consider as an example the scheme in Fig. 4(a). First we look at the left side of the cavity, in which frequencies were increased at EOM1 in the QW on the line. With $\overline{E O M}$ of Fig. 6 replacing EOM1 in Fig.4(a), consider any step at which the field entering $\overline{E O M}$ contains all the allowed frequencies from $\omega-M \bar{\omega}$ to $\omega+M \bar{\omega}$. The role of M1 is to separate the frequency $\omega+M \bar{\omega}$, which is directed to EOMa, from the rest of frequencies, which are directed to EOMb. The modulators are configured such that EOMa decreases the entering frequency by $2 M \bar{\omega}$, while EOMb increases 
Figure 6: Scheme of the $\overline{E O M}$ device that substitutes the electrooptic modulators in Fig.3(a) in order to perform the QW on the circle. M1 and M2 are mirrors that reflect a single frequency $(M \bar{\omega}$ and $-M \bar{\omega}$, respectively) and EOMa and EOMb are electrooptic modulators. See text for a detailed description of the device operation.

the frequencies entering it in $\bar{\omega}$. After traversing the electrooptic modulators, the frequencies enter M2. The frequencies $\{\omega-(M-1) \bar{\omega}, \ldots, \omega+M \bar{\omega}\}$ that come from EOMb traverse M2, while the frequency $\omega-M \bar{\omega}$, that comes from EOMa is reflected by M2. Then, the set of frequencies exiting $\overline{E O M}$ is the same set that entered, but all of them have been shifted appropriately.

In the right side of the cavity in Fig.4(a), where frequencies were decreased in the QW on the line, EOM2 is substituted by another $\overline{E O M}$ that works in a slightly different way to the one in the left side of the cavity: first, the positions of M2 and M1 are interchanged (or, what is the same, light enters from below), and second, EOMa and EOMb in $\overline{E O M}$ are modified in the sense that EOMa now increases the incoming frequency in $2 M \bar{\omega}$ whilst EOMb decreases in $\bar{\omega}$ the rest of frequencies. In this way, in the right side of the cavity all frequencies entering $\overline{E O M}$ are decreased except $\omega-M \bar{\omega}$ that is converted into $\omega+M \bar{\omega}$ as it must be. Of course it is essential that the optical lengths of the two paths in $\overline{E O M}$ be symmetric in order not to introduce any spurious dephasing during the separation of frequencies.

We finally comment that the device $\overline{E O M}$ can be also used in the cavities of Figs.3(a) and 4(b) by suitably adapting its operation. In particular, in the scheme of Fig.4(b) a single $\overline{E O M}$ is sufficient for performing the QW on the circle: as the clockwise and counterclockwise fields have orthogonal polarizations, the electrooptic modulators in $\overline{E O M}$ can increase (decrease) the frequencies oppositely for the two polarization components.

\section{Discussion}

We have shown in detail how quantum walks, both on the line and on the circle, can be implemented in optical cavities. We have also discussed the close relation existing between the optical Galton board of Bouwmeester et al. 29] and the QW on the line. In fact we have shown that the experiment of [29] can be 
regarded as a proof of principle that our proposals for the implementation of the QW can be carried out in optical cavities. All of this leads to the obvious conclusion, as noted earlier by us [28], that the coined QW does not require quantization for its implementation and thus, in this sense, it is not quantum. Nevertheless this point requires further discussion.

First of all we should carefully distinguish between an actual implementation and a simulation of the QW. A simulation would not capture all the characteristics of the QW whilst an implementation would do. Then, in order to make an assesment as strong as "the QW is not quantum but classical" we must be completely sure that what we are proposing is an implementation and not a simulation as in this last case some quantum features would be lost an this would manifest in some measurable characteristics. In order to make this distinction clearer, let us consider the optical implementations of the Grover's search algorithm [3, 4]: it is rather clear that these cannot be regarded as actual implementations but only as simulations as the lack of quantum entanglement manifests in the exponential increase of classical resources with the increase of the problem size. In other words, with the only use of classical resources, quantum entanglement can be simulated but not implemented and this manifests in the scaling of the needed resources. Then, our proposal is an implementation or a simulation?

In order to answer this question we should first clarify the role played by entanglement in the QW, something that is beyond the scope of this article and that we plan to investigate elsewhere, but even without this knowledge we can still clarify to some extent this point. It is obvious that the classical resources needed in the schemes here proposed for the implementation of the QW on the line (or on the circle) do not grow with the number of steps in the QW except, perhaps, in the detection stage but here the resources needed would grow linearly with the number of steps, as would grow in a quantum implementation. Then it seems that entanglement does not play any role in the QW and we can consider our proposals as actual implementations. But this first conclussion could be misleading as the increase in the problem size does not lie in the number of steps in the walk but in the dimension of the QW. We have noted above that our schemes could be generalized for implementing multidimensional QWs [13: for that, coins with more than two states are needed or, in other words, several coins are needed. Polarization and direction cebits could be used simultaneously for implementing these multidimensional QWs. It would be here where the problem of the scaling of the classical resources would appear: a $d$ dimensional $\mathrm{QW}$ requires $d$ coin-qubits but their classical simulation requires $2^{d}$ light beams [1]. Then, it is in these higher dimensional walks that quantum entanglement would manifest. In conclussion: one-dimensional (even two-dimensional) QWs can be classically implemented but higher dimensional QWs can only be simulated. Of course this is a very preliminary conclussion as in some cases multidimensional QWs could be reduced to equivalent unidimensional QWs [21.

Let us finally note that one of the interesting phenomena that could be studied by using any of the designs here proposed is the effect of decoherence on the QW. It has been shown by Kendon and Tregenna 16] that decoherence can 
be beneficial for QWs in the sense that it can lead, under suitable conditions, to more homogeneous probability distributions. Decoherence can act on the walker and/or on the coin, and it could be simulated in the schemes devised here by introducing, e.g., random changes in the polarization or direction of the light beams.

\section{Acknowledgements}

This work has been supported in part by the UK Engineering and Physical Sciences Research Council and the European Union. ER acknowledges a grant from the Ministerio de Educación, Cultura y Deportes of the Spanish Goverment (Grant PR20002-0244) and partial finantial support from the spanish Ministerio de Ciencia y Tecnología and European FEDER funds through project BFM200204369-C04-01. JES acknowledges financial support from the Natural Sciences and Engineering Research Council of Canada. We gratefully acknowledge Dr. Javier García (Universitat de València, Spain) for his careful reading of the manuscript.

\section{References}

[1] R.J.C. Spreeuw, Phys. Rev. A 63, 062302 (2001)

[2] N.J. Cerf, C. Adami, and P.G. Kwiat, Phys. Rev. A 57, R1477 (1998)

[3] P.G. Kwiat, J.R. Mitchell, P.D.D. Schwindt, and A.G. White, J. Mod. Opt. 47, 157 (2000)

[4] N. Bhattacharya, H.B. van Linden van den Heuvell, and R.J.C. Spreeuw, Phys. Rev. Lett. 88, 137901 (2002)

[5] Y. Aharonov, L. Davidovich, and N. Zagury, Phys. rev. A 48, 1687 (1993)

[6] D. Meyer, J. Stat. Phys. 85, 551 (1996)

[7] E. Farhi and S. Gutman, Phys. Rev. A 58, 915 (1998)

[8] A. Nayak, and A. Vishwanath, e-print quant-ph/0010117

[9] D. Aharonov, A. Ambainis, J. Kempe, and U. Vazirani, Proceedings of the 30th Anual ACM Symposium on Theory of Computation (ACM Press, New York, 2001) 50; e-print quant-ph/0012090

[10] J. Kempe, e-print quant-ph/0205083

[11] N. Konno, Quantum Information Processing 1, 345 (2002); e-print quant-ph/0206103

[12] E. Bach, S. Coppersmith, M.P. Goldschen, R. Joynt, and J. Watrous, eprint quant-ph/0207008 
[13] T.D. Mackay, S.D. Barltett, L.T. Stephenson, and B.C. Sanders, J. Phys. A: Math. Gen. 35, 2745 (2002)

[14] N. Shenvi, J. Kempe, and K.B. Whaley, Phys. Rev. A 67, 052307 (2003)

[15] A.M. Childs, R. Cleve, E. Deotto, E. Farhi, S. Gutman, and D. A. Spielman, quant-ph/0209131

[16] V. Kendon and B. Tregenna, Phys. Rev. A 67, 042315 (2003)

[17] M. Bednarska, A. Grudka, P. Kurzynski, T. Luczak, and A. Wojcik, e-print quant-ph/0304113

[18] M. Hillery, J. Bergou, and E. Feldman, e-print quant-ph/0302161

[19] H.A. Carteret, M.E.H. Ismail, and B. Richmond, e-print quant-ph/0303105

[20] B. Tregenna, W. Flanagan, R. Maile, and V. Kendon, e-print quant-ph/0304204

[21] J. Kempe, Contemp. Phys. 44, 307 (2003)

[22] We thank Steven Bartlett, of Macquarie University, for pointing this out to us. See, for example, C.H. Papadimitriou, Computational Complexity (Addison Wesley, 1994)

[23] B.C. Travaglione and G.J. Milburn, Phys. Rev. A 65, 032310 (2002)

[24] W. Dür, R. Raussendorf, V.M. Kendon, and H.-J. Briegel, Phys. Rev. A 66, $052319(2002)$

[25] B.C. Sanders, S.D. Bartlett, B. Tregenna, and P.L. Knight, Phys. Rev. A 67, 042305 (2003)

[26] Z. Zhao, J. Lu, H. Li, T. Yang, Z.-B Chen, and J.-W. Pan, e-print quant-ph/0212149

[27] H. Jeong, M. Paternostro, and M.S. Kim, e-print quant-ph/0305008

[28] P.L. Knight, E. Roldán, and J.E. Sipe, Phys. Rev. A 68, 020301 (2003)

[29] D. Bouwmeester, I. Marzoli, G.P. Karman, W. Schleich, and J.P. Woerdman, Phys. Rev. A 61, 013410 (2000) 


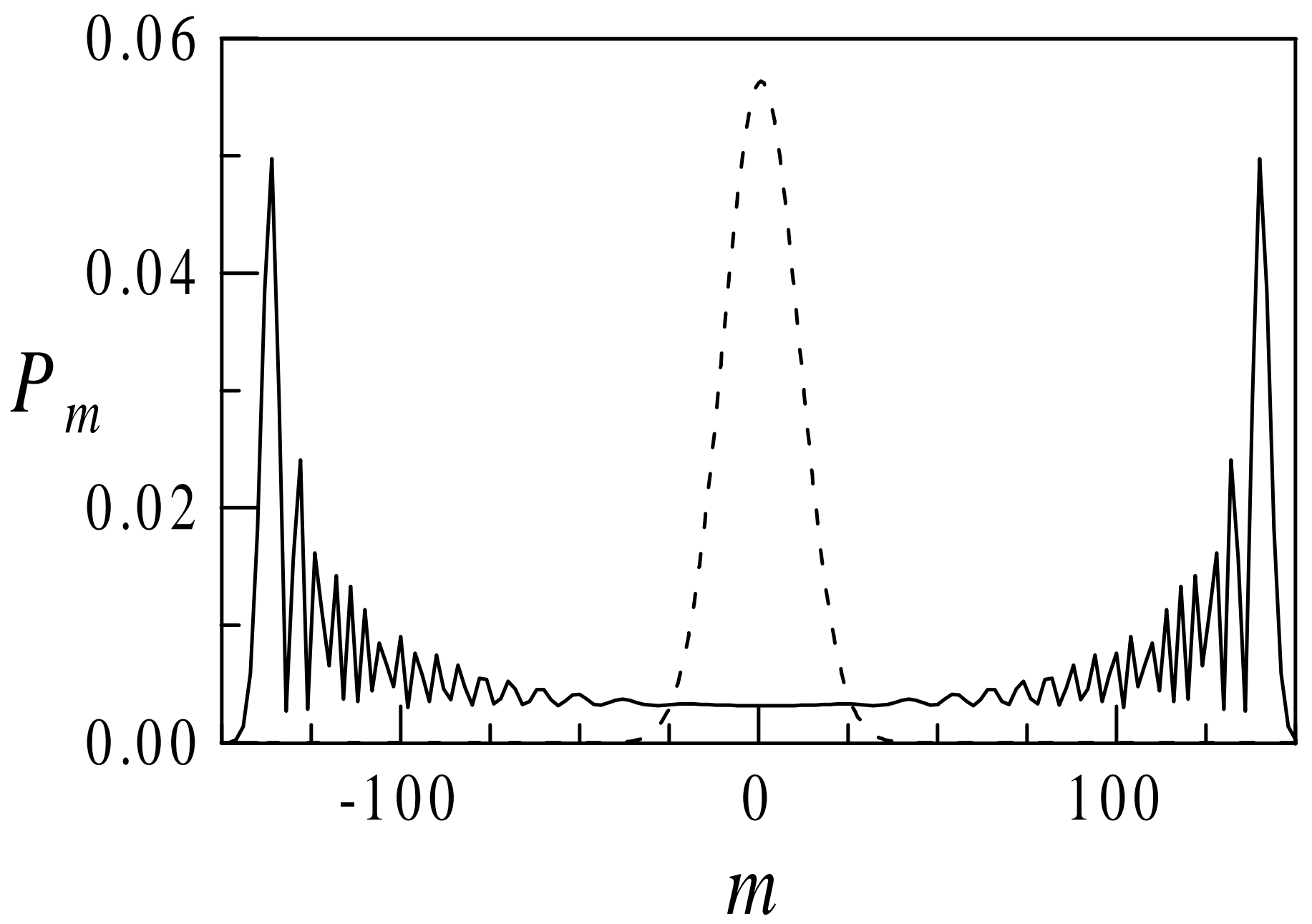




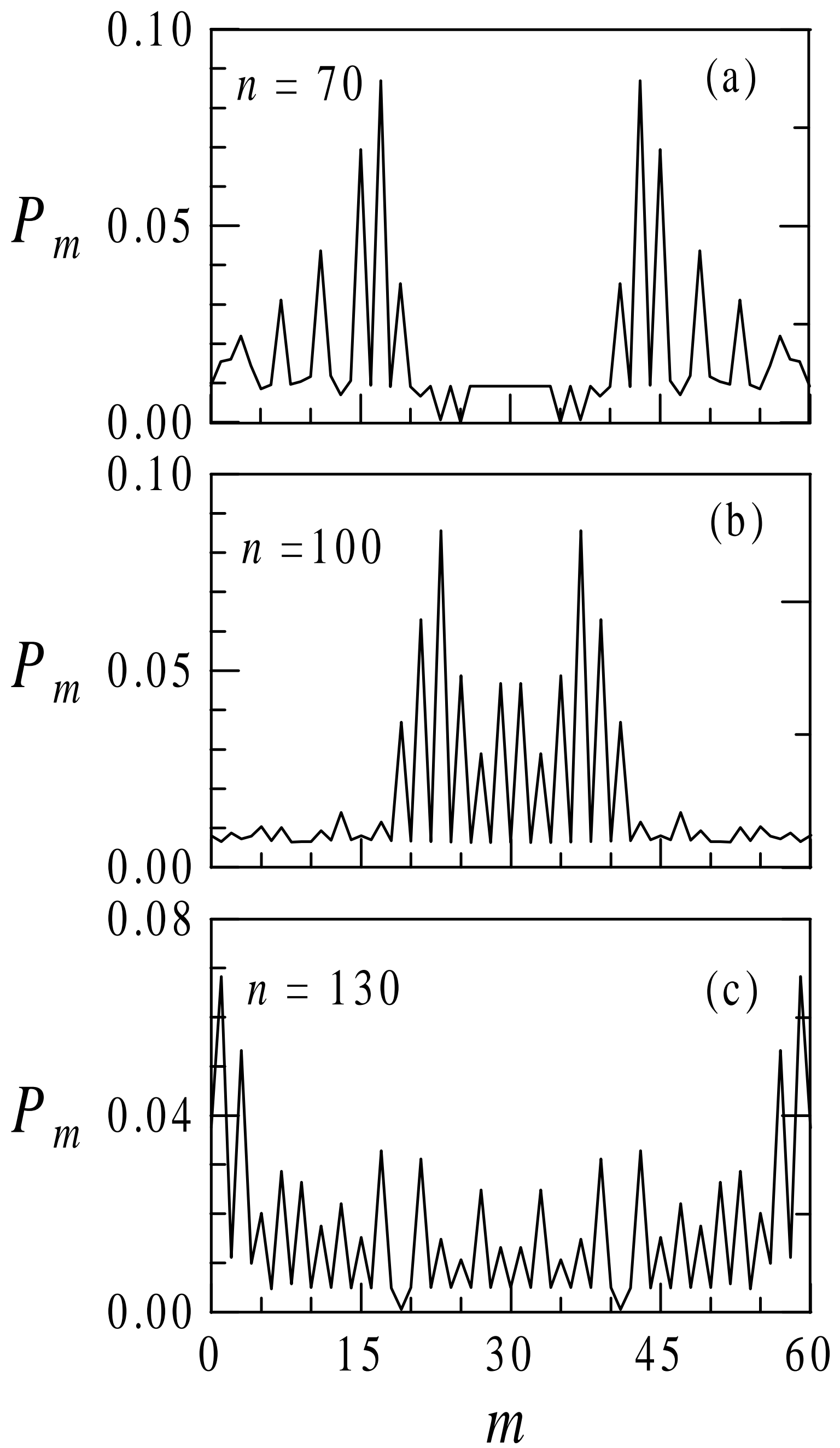




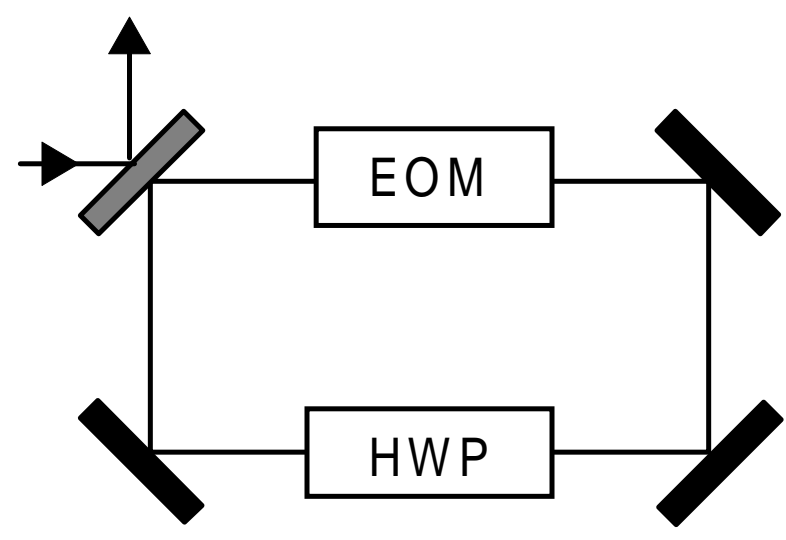

(a)

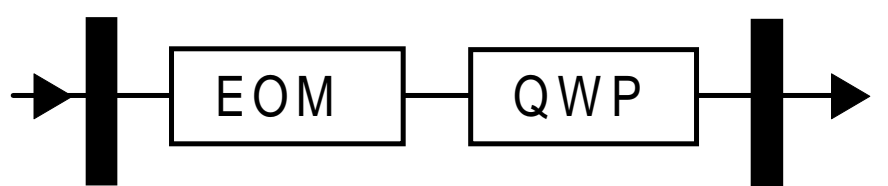

(b) 
(a)
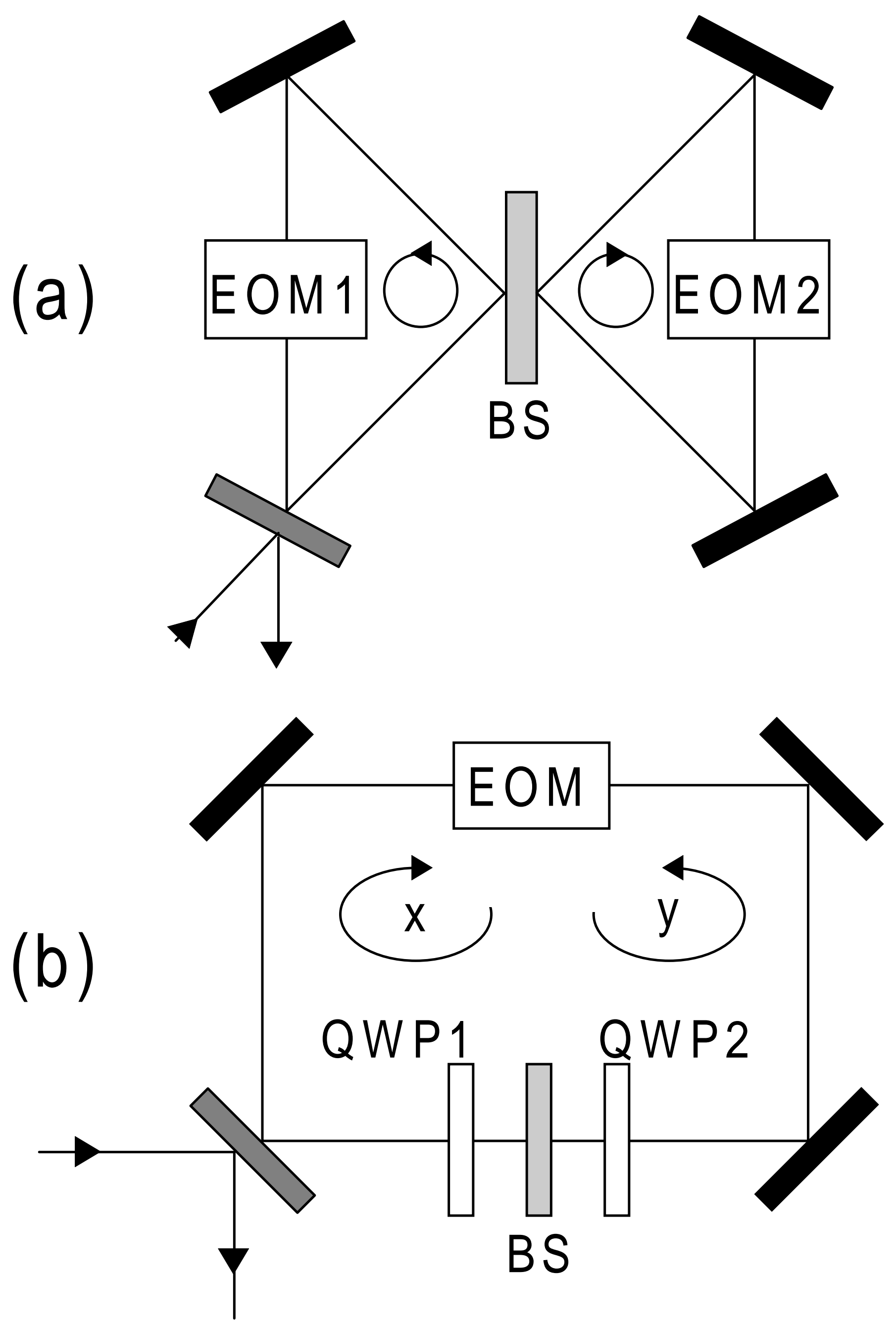

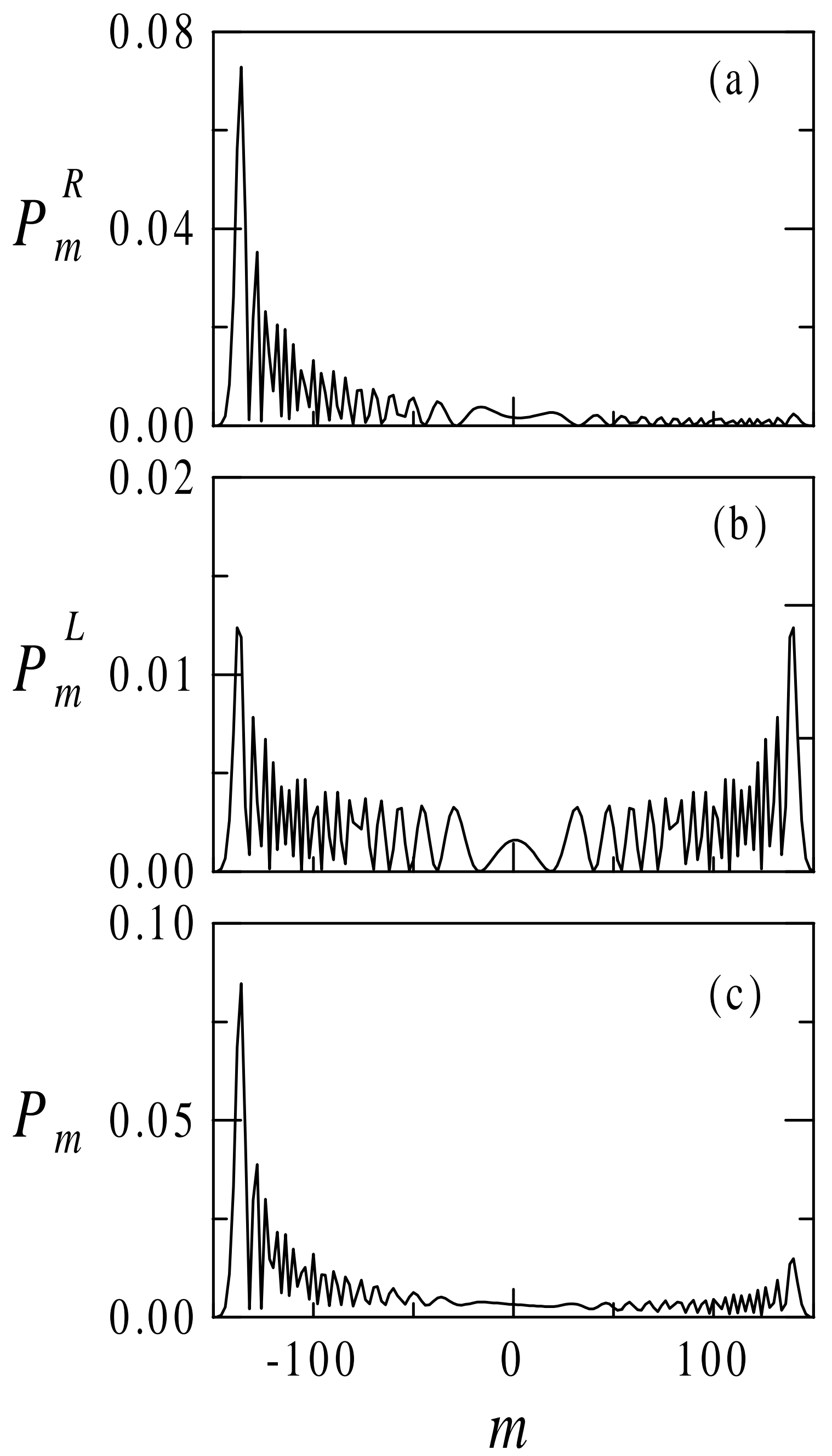


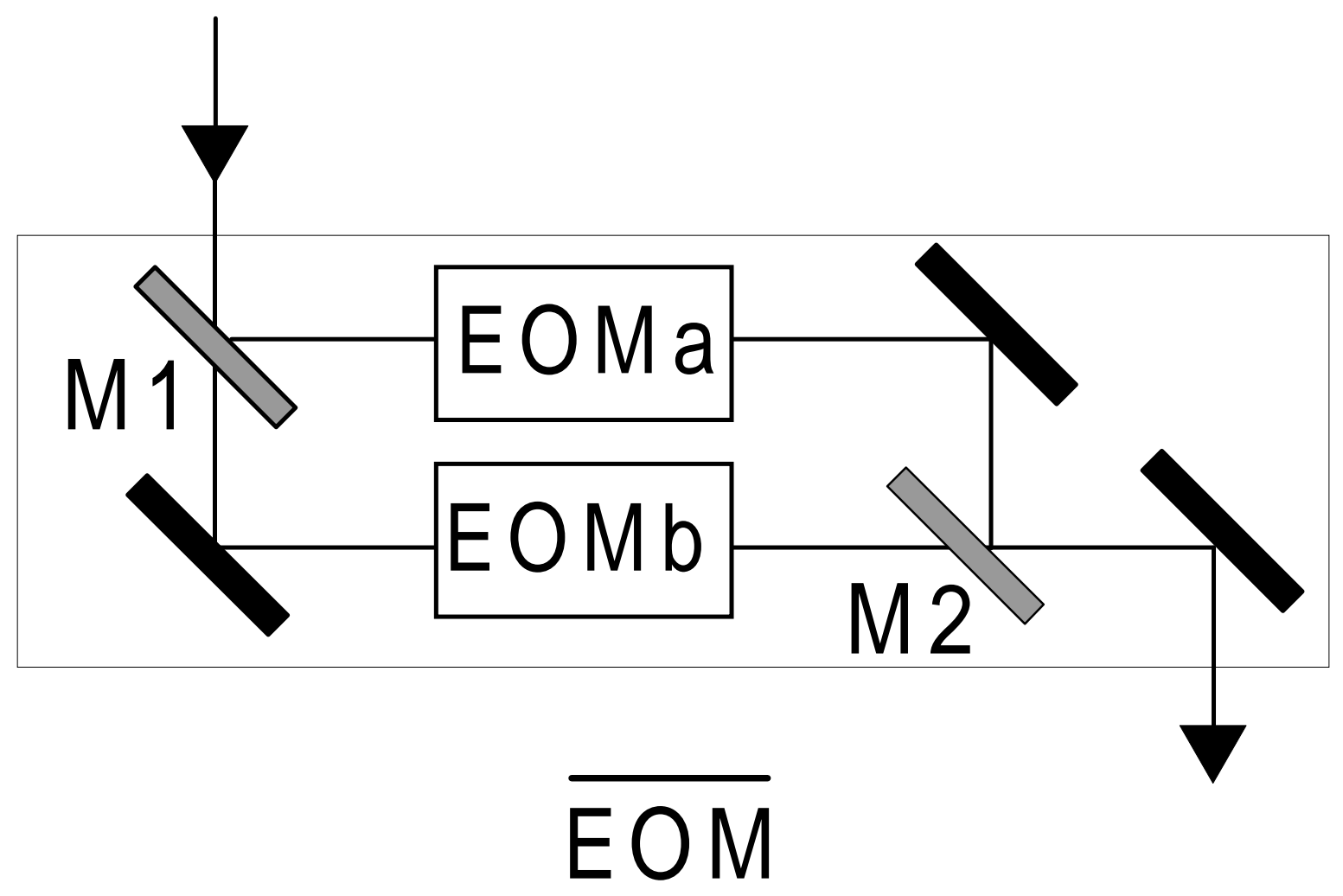

